\title{
Kiwifruit consumption favourably affects plasma lipids in a randomised controlled trial in hypercholesterolaemic men
}

\author{
Cheryl S. Gammon ${ }^{1 *}$, Rozanne Kruger ${ }^{1}$, Anne M. Minihane ${ }^{2}$, Cathryn A. Conlon ${ }^{1}$, Pamela R. von Hurst ${ }^{1}$ \\ and Welma Stonehouse ${ }^{1}$ \\ ${ }^{1}$ Institute of Food, Nutrition and Human Health, Massey University, Albany Campus, Private Bag 102904, Auckland 0745, \\ New Zealand \\ ${ }^{2}$ Department of Nutrition, Norwich Medical School, University of East Anglia, Norwich NR4 7TJ, UK
}

(Submitted 25 May 2012 - Final revision received 9 August 2012 - Accepted 27 August 2012 - First published online 14 November 2012)

\begin{abstract}
The unique composition of green kiwifruit has the potential to benefit CVD risk. The aim of the present study was to investigate the effect of consuming two green kiwifruits daily in conjunction with a healthy diet on plasma lipids and other metabolic markers and to examine response according to $A P O E$ genotype in hypercholesterolaemic men. After undergoing a 4-week healthy diet, eighty-five hypercholesterolaemic men (LDL-cholesterol (LDL-C) $>3.0 \mathrm{mmol} / \mathrm{l}$ and TAG $<3 \mathrm{mmol} / \mathrm{l}$ ) completed an 8-week randomised controlled cross-over study of two 4-week intervention sequences of two green kiwifruits per d plus healthy diet (intervention) or healthy diet alone (control). Anthropometric measures, blood pressure (BP) and fasting blood samples (plasma lipids, serum apoA1 and apoB, insulin, glucose, high-sensitivity C-reactive protein (hs-CRP)) were taken at baseline, and at 4 and 8 weeks. After the kiwifruit intervention, plasma HDLcholesterol (HDL-C) concentrations were significantly higher (mean difference $0 \cdot 04 ; 95 \%$ CI $0 \cdot 01,0 \cdot 07 \mathrm{mmol} / 1 ; P=0 \cdot 004$ ) and the total cholesterol (TC):HDL-C ratio was significantly lower (mean difference $-0 \cdot 15 ; 95 \%$ CI $-0 \cdot 24,-0 \cdot 05 \mathrm{mmol} / 1 ; P=0 \cdot 002$ ) compared with the control. In carriers of the APOE4 allele, TAG decreased significantly (mean difference $-0 \cdot 18 ; 95 \% \mathrm{CI}-0 \cdot 34,-0 \cdot 02 \mathrm{mmol} / \mathrm{l}$; $P=0.03)$ with kiwifruit compared with control. There were no significant differences between the two interventions for plasma TC, LDL-C, insulin, glucose, hs-CRP and BP. The small but significant increase in HDL-C and decrease in TC:HDL-C ratio and TAG (in $A P O E 4$ carriers) suggest that the regular inclusion of green kiwifruit as part of a healthy diet may be beneficial in improving the lipid profiles of men with high cholesterol.
\end{abstract}

Key words: Kiwifruit: CVD: Lipids: ApoE

In New Zealand (NZ), despite a decline of over $60 \%$ in CVD mortality rates since its peak in the late 1960s, CVD remains one of the leading causes of death, contributing to over $28 \%$ of overall mortality ${ }^{(1,2)}$. While pharmacological interventions have made a substantial contribution to the decline in incidence since the 1990s, it is speculated that changes in dietary consumption behaviours may have made the biggest contribution before then ${ }^{(3)}$.

One of the changes in dietary patterns has been a steady increase in fruit consumption, but $45 \%$ of $\mathrm{NZ}$ males still do not consume the recommended number of two fruit servings per $\mathrm{d}^{(4)}$. Fruits are chemically complex foods that contain a range of nutrient and non-nutrient components that may contribute independently or synergistically to cardiovascular health $^{(5-7)}$. These include soluble fibre, antioxidant vitamins, folate and phytochemicals ${ }^{(5,6,8-10)}$.
In NZ, kiwifruit is a commonly consumed, nutrient-dense fruit. Green kiwifruits are unique, in that they contain significant levels of soluble fibre ${ }^{(11)}$ and have one of the highest concentrations of vitamin $\mathrm{C}$ of any readily available fruit $^{(12)}$. Kiwifruit has been shown to be a significantly better delivery vehicle to replenish depleted vitamin $\mathrm{C}$ tissue levels than a supplement, in a mouse model ${ }^{(13)}$. Green kiwifruit also contains a significant amount of vitamin $\mathrm{E}$, which is more commonly associated with green leafy vegetables than fruit (other than avocados), K, folate and other phytochemicals, including polyphenols and carotenoids ${ }^{(12,14)}$. Many of the phenolics and flavonoids are yet to be identified, as to date they have been unextractable ${ }^{(15)}$. Few studies have investigated the effects of kiwifruit on CVD-related metabolic markers. Two previous studies investigating their effects on lipid profiles have shown conflicting results, and neither study

Abbreviations: Baseline 1, before nutrition consultation (visit 1); Baseline 2, after completing 4-week healthy diet run-in (visit 3); HDL-C, HDL-cholesterol; HOMA, homeostasis model assessment; hs-CRP, high-sensitivity C-reactive protein; LDL-C, LDL-cholesterol; NZ, New Zealand; sLDL, small dense LDL; TC, total cholesterol. 
included a control group ${ }^{(16,17)}$. A recent study has shown beneficial effects on blood pressure ${ }^{(18)}$.

Genetic variation is known not only to contribute to $>45 \%$ of CVD risk ${ }^{(19)}$, but also to explain a large component of the highly heterogeneous response to interventions ${ }^{(20,21)}$. An example is the common $A P O E$ epsilon variant, where, in a general population, up to a $50 \%$ higher risk of CVD is associated with the APOE4 compared with the wild-type $A P O E 3 / E 3$ genotype $^{(22)}$. Although recent evidence is suggestive of a pro-inflammatory and pro-oxidant impact of the APOE4 allele $^{(23)}$, the higher risk has been traditionally attributed to higher total cholesterol (TC) and LDL-cholesterol (LDL-C) concentrations in this subgroup ${ }^{(24)}$. Furthermore, although the literature is inconsistent, carriers of the APOE4 allele appear to show greater LDL-C-lowering responses in dietary fat manipulation studies ${ }^{(25)}$.

We hypothesised that the replacement of two fruit servings in a healthy diet with two green kiwifruits would have favourable effects on plasma lipids and other CVD-related markers of risk, and that carriers of different $A P O E$ genotypes may show differences in response to the intervention. We tested this hypothesis using a randomised controlled cross-over study design comparing a healthy diet including kiwifruit with a healthy diet with no kiwifruit. In addition to having a control group, unlike other dietary intervention studies of kiwifruit, the present study is the first to examine the beneficial impact of kiwifruit in addition to general recommended healthy eating guidelines. Thus, the beneficial effects of kiwifruit beyond the effects of a healthy diet containing fruit could be investigated.

\section{Subjects and methods}

\section{Subjects}

A total of eighty-seven men over 21 years of age were recruited from around Auckland, NZ, through a range of advertising strategies (emails to selected databases, such as the Massey University Albany Nutrition Research volunteer database, press releases and fliers). To be included in the study, the subjects needed to have a LDL-C concentration $>3.0 \mathrm{mmol} / \mathrm{l}$ and a plasma TAG concentration $<3.0 \mathrm{mmol} / \mathrm{l}$. Exclusion criteria included diagnosed chronic disease such as CHD, diabetes and cancer; smoking; and the use of any product that could affect serum lipid concentrations, including lipid-lowering medication (statins, fibrates and cholesterol absorption inhibitors), supplements such as nicotinic acid, fish oil capsules, fibre-containing laxatives or functional foods such as sterol-enriched spreads. Additionally, subjects were excluded if familial hypercholesterolaemia was determined using the diagnostic criteria for familial hypercholesterolaemia described in de Sauvage Nolting et al. ${ }^{(26)}$.

As one of the objectives of the study was to compare the effects of the intervention between carriers of the APOE4 allele and non-carriers, it was calculated that a minimum of eighty subjects would be required to ensure a sample size of twenty carrying the APOE 4 allele. This was based on retrospective genotyping and an estimated prevalence of $25 \%$ in
Caucasian populations ${ }^{(23)}$. A sample size of twenty APOE4 carriers would provide $80 \%$ power to detect a significant difference ( $\alpha=0.05$, two-tail) of $0.5 \mathrm{mmol} / \mathrm{l}( \pm 7-10 \%)$ in TC and LDL-C between interventions. The power calculation was based on the evidence that a reduction of $0.5 \mathrm{mmol} / 1 \mathrm{LDL}-\mathrm{C}$ is estimated to result in a $12-14 \%$ reduction in CHD mortality risk, which is a clinically significant reduction ${ }^{(27)}$. More subjects ( $n$ 87) were recruited to allow for drop-outs from the study.

The present study was conducted according to the guidelines laid down in the Declaration of Helsinki, and all procedures involving human subjects were approved by the Massey University Human Ethics Committee: Southern A 09/76. Written informed consent was obtained from all subjects. Clinical trial registry: ACTRN12610000213044 www.ANZCTR.org.au

All subjects received nominal compensation for travel expenses and, at completion of the study, subjects received reports summarising their individual results including body composition, BP, blood results and dietary assessment, and additional dietary recommendations were provided.

\section{Study design}

The study was a randomised controlled cross-over study (Fig. 1). After completion of a 4-week run-in on a healthy diet, subjects were randomly assigned to one of two groups (dietary intervention sequences) using computer-generated random numbers (http://www.randomization.com). The study consisted of two 4-week intervention sequences of two green kiwifruits per d plus healthy diet (intervention) or healthy diet alone (control). The study was conducted between May and September 2010.

Subjects made five visits to the Massey University Human Nutrition Research Unit; baseline 1 - before their nutrition consultation (visit 1), at the time of their nutrition consultation (visit 2), baseline 2 - after completing their 4-week healthy diet run-in (visit 3) and at the end of each intervention (visits 4 and 5). Anthropometric measures, fasting blood samples and dietary data were gathered at all visits (excluding the nutrition consultation visit), as described later.

The primary outcome measures were lipid profiles (plasma TC, LDL-C, TAG, HDL-cholesterol (HDL-C), TC:HDL-C ratio, serum apoA1 and apoB) and small dense LDL (sLDL). The secondary outcome measures were markers of glucose metabolism (plasma glucose, serum insulin), blood pressure and serum high-sensitivity C-reactive protein (hs-CRP).

The study was conducted under free-living conditions. The subjects were asked to maintain their normal daily routine (including physical activity) while implementing the healthy diet guidelines, for the duration of the study. Compliance with these instructions, following the intervention, presence of illness and use of medication during the study was monitored by a weekly self-completed diary.

\section{Diet and kiwifruit interventions}

The healthy diet was based on the Heart Foundation of New Zealand's '9 steps to Eating for a Healthy Heart' (see Appendix 1 of the Supplementary material, available 


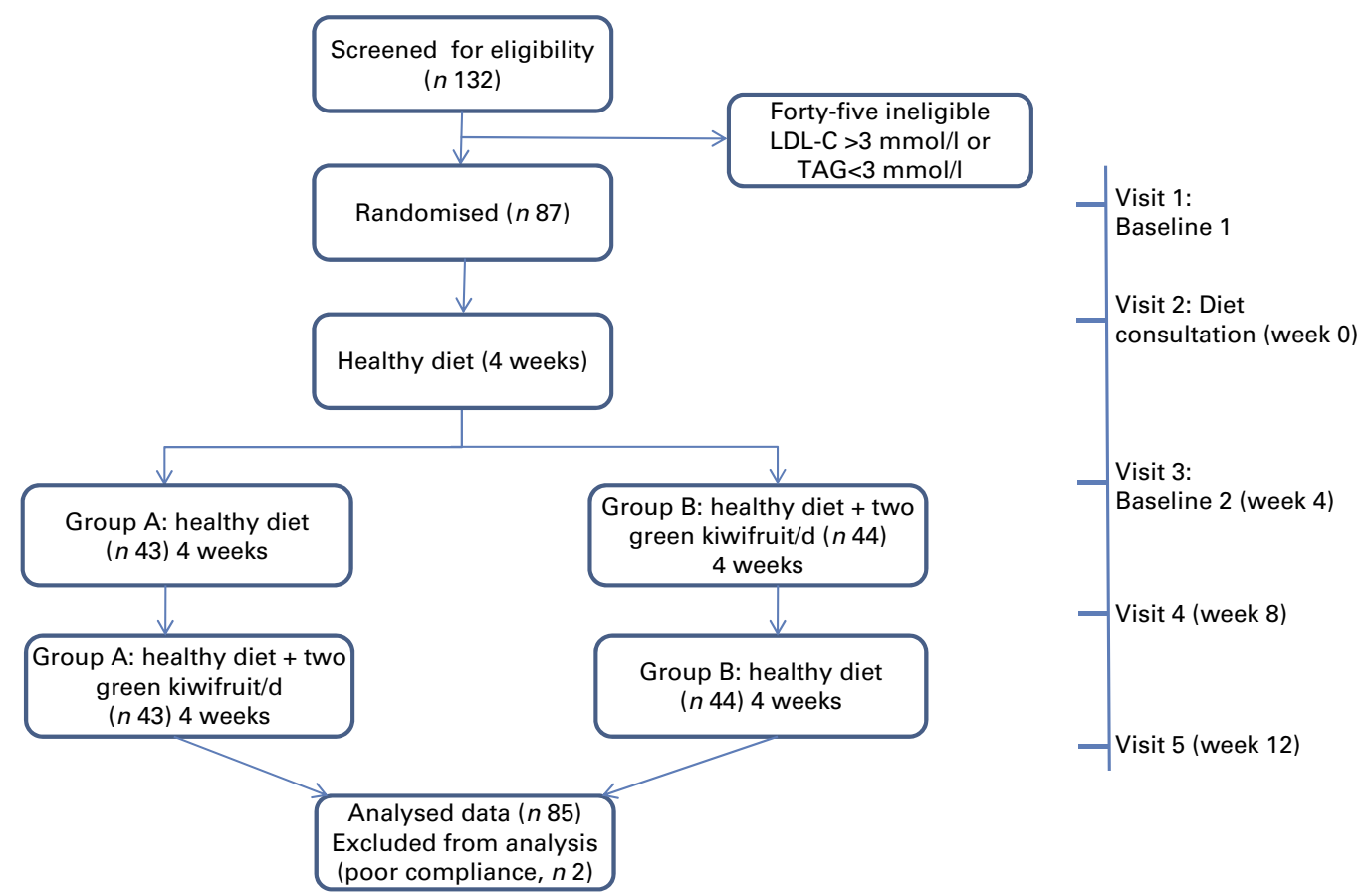

Fig. 1. Study flow (A colour version of this figure can be found online at http://www.journals.cambridge.org/bjn).

online $)^{(28)}$. All subjects were required to attend a nutrition consultation with a nutritionist, who outlined the guidelines and suggested personal changes to each subject's present diet. Subjects were then requested to follow the healthy diet (control diet) for the 12 weeks of the study. The guidelines required subjects to consume at least two servings of fruits per $\mathrm{d}$, and during the kiwifruit intervention, two of these servings were replaced with two Zespri ${ }^{\circledR}$ green kiwifruits (Actinidia deliciosa var Hayward) per d.

Before their nutrition consultation, subjects were required to complete a $3 \mathrm{~d}$ food record, and $24 \mathrm{~h}$ food records were collected at visits 3-5. During each visit, subjects met with a nutritionist, who instructed them on how to complete food records, checked the records for completeness and portion size accuracy and completed a quality-control checklist based on the subjects' achievement of the 'healthy diet' guidelines. A questionnaire to assess how subjects tolerated consuming two green kiwifruits per $d$ was completed at the end of the kiwifruit intervention.

Nutrient analysis for the $3 \mathrm{~d}$ food record and subsequent $24 \mathrm{~h}$ food records was conducted using the computer program FoodWorks Professional Edition version 4.00 (Xyris Software, 2009). The three $24 \mathrm{~h}$ food records were combined to give an average dietary intake over the 8-week intervention period, which allowed comparison with the $3 \mathrm{~d}$ food record.

\section{Anthropometric measurements}

Height (visit 1 only) and waist and hip circumferences were measured at visits 1 and 3-5 using the International Society for the Advancement of Kinanthropometry anthropometry methods using a stadiometer and Lufkin tape. Weight and percentage body fat were measured using the BODPOD (air displacement plethysmography, COSMED USA, Inc.). BMI and waist:hip ratio were then calculated.

\section{Blood pressure}

An Omron HEM-907 Digital Automatic Blood Pressure Monitor (Omron Healthcare, Inc.) was used to measure brachial arterial BP. Subjects had to lie down quietly to relax for about $5 \mathrm{~min}$ before any measurements were taken. The right arm was used for all measurements. Two measurements were taken consecutively, $1 \mathrm{~min}$ apart, and the average calculated using the auto function of the monitor.

\section{Sample collection and preparation}

Venous blood samples were collected after fasting for $8 \mathrm{~h}$ into vacutainers buffered with EDTA, heparin or no anticoagulant (baseline 1, heparin vacutainers only, for plasma TC, TAG and HDL-C).

Serum samples were allowed to clot for $30 \mathrm{~min}$ before being processed. All other tubes were kept on ice and processed within $60 \mathrm{~min}$ of collection. Tubes were centrifuged for $15 \mathrm{~min}$ at $3000 \mathrm{rpm}$ at $4^{\circ} \mathrm{C}$. Aliquots were stored at $-80^{\circ} \mathrm{C}$ until the end of the study for analysis in one batch.

\section{Biochemical analysis}

Analysis was conducted by the Canterbury Health Laboratories, Christchurch, NZ (IANZ ISO 15189) for all samples except the sLDL, which was analysed at the Biomedical Research Centre, University of East Anglia. Plasma TC and TAG concentrations were measured with enzymatic kits (Abbott Laboratories, catalogue no. 7D62-21 and 
catalogue no. 7D74-21, respectively). HDL-C was measured by accelerated reaction using selective detergent (Abbott Laboratories, Catalogue no. 3K33-21). LDL-C was calculated using the Friedewald algorithm (LDL-C $=$ TC $-\mathrm{HDL}-\mathrm{C}-(\mathrm{TAG}$ ) $2.22) \mathrm{mmol} / \mathrm{l})^{(29)}$. The interassay $\mathrm{CV}$ ranged from 1.7 to $2.0 \%$ for total plasma cholesterol, 2.5 to $5.9 \%$ for plasma TAG and 3.7 to $10 \cdot 1 \%$ for plasma HDL-C. Serum apoA1 and apoB were measured using Abbott Architect/Aeroset reagent kits (Abbott Laboratories, Catalogue no. 9D92-20 and catalogue no. 9D93-20, respectively), CV 3 and 4\%, respectively.

Plasma glucose was determined by enzymatic assay (Abbott Laboratories, Catalogue no. 3L82-41), CV 1.72-2.49\%, and plasma insulin by two-site sandwich after polyethylene glycol extraction (Roche, Catalogue no. 12017547), CV 3.8-8.7\%. The homeostasis model assessment (HOMA) 2 model was used to calculate insulin resistance, based on fasting insulin and glucose concentrations ${ }^{(30)}$.

Serum hs-CRP was measured by Nephelometry assay (Siemens Healthcare Diagnostic Products $\mathrm{GmbH}$, Catalogue no. 10446091), CV 4.5-5.1\%. APOE variants were genotyped by $\mathrm{PCR}^{(31)}$. sLDL-C was measured by direct automated assay, sLDL-Ex 'SEIKEN' two-reagent kit (Randox Laboratories, Catalogue no. 56216), intra-assay CV 0.99\% and inter-assay CV $1.24 \%$.

\section{Nutrient analysis of kiwifruit samples}

Kiwifruit samples were randomly selected from each batch (one batch per 4-week period) of kiwifruit and allowed to ripen at room temperature to the same level of ripeness as recommended to the subjects for consumption. Fruit was then peeled, sliced, vacuum packed and frozen before being freeze-dried. The macronutrients, vitamin $\mathrm{C}$, vitamin $\mathrm{E}$ and dietary fibre (insoluble and soluble) were analysed as follows: protein - Leco, total combustion method, AOAC 968.06; fat - cold extraction using chloroform-methanol, AOAC 969.24; carbohydrate - by difference; sugars - phenol sulphuric $^{(32)}$; vitamin C - HPLC ${ }^{(33)}$; vitamin E - AOAC Official methods 971.30, modified and dietary fibre - Megazyme total dietary fibre kit (Megazyme International Ireland Limited), method details: AOAC 991.43, AOAC 985.29, AACC32-07 and AACC 32-05.

\section{Statistical analysis}

Statistical analyses were performed using SPSS software version 18 (SPSS, Inc.). Normal distributions were tested using the Kolmogorov-Smirnov and Shapiro-Wilk tests and

Table 1. Baseline characteristics of subjects

(Mean values and $95 \%$ confidence intervals (at baseline 1, unless otherwise indicated); geometric means, $95 \%$ confidence intervals, percentages and ranges; $n$ 85)

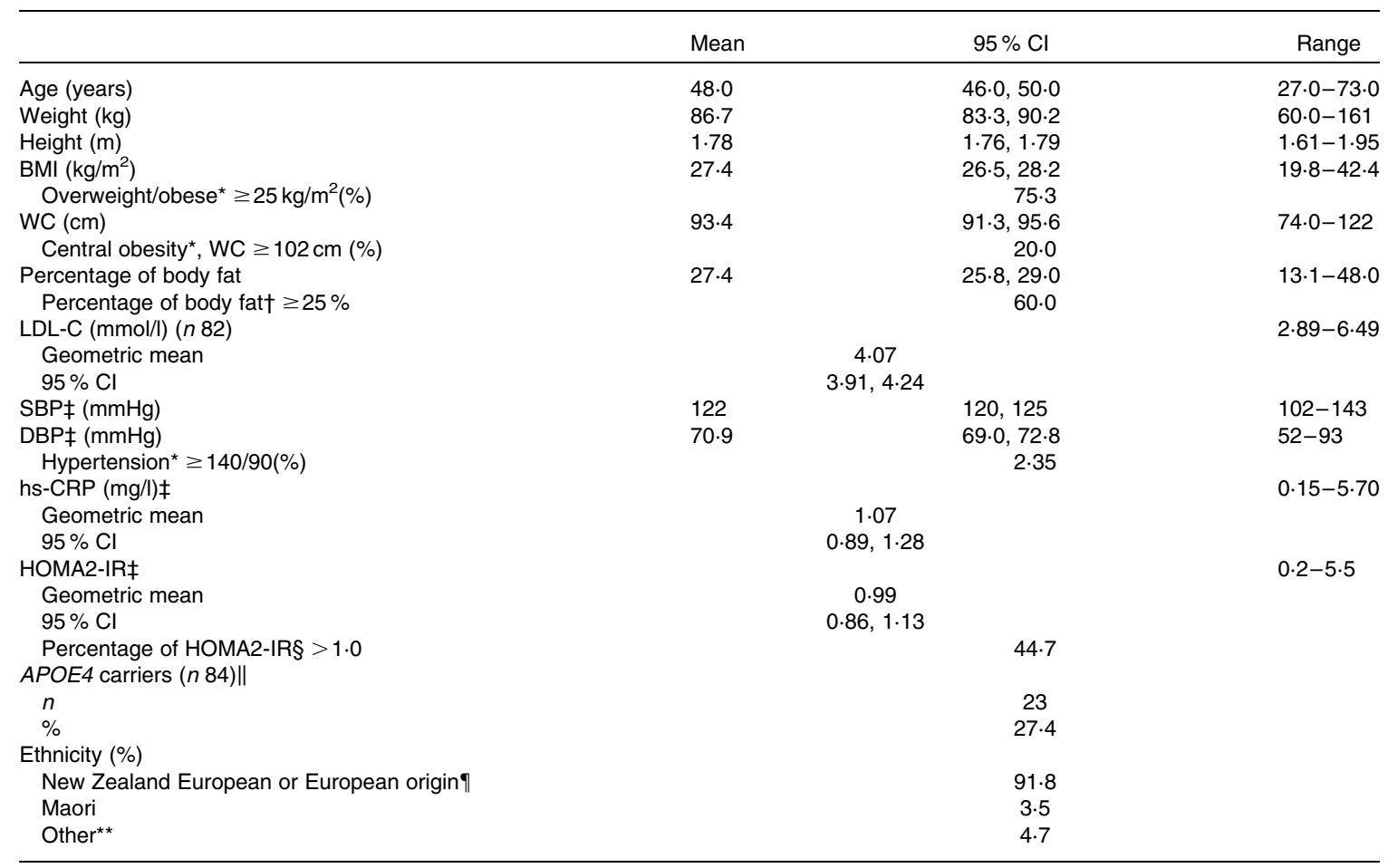

WC, waist circumference; LDL-C, LDL-cholesterol; SBP, systolic blood pressure; DBP, diastolic blood pressure; hs-CRP, high-sensitivity C-reactive protein; HOMA2-IR, homeostasis model assessment 2 model for insulin resistance.

${ }^{*}$ Recognised cut-offs for WC, BMI and hypertension ${ }^{(56)}$.

$\dagger$ Recognised cut-off for excess body fat ${ }^{(34,35)}$.

$\ddagger$ Baseline 2 values.

$\S$ Elevated insulin resistance ${ }^{(30)}$.

\| One subject requested not to be included in any gene analysis.

ๆ European origin: United Kingdom, South Africa, Europe and Australia.

${ }^{\star \star}$ Chinese, Philippines, Tonga and India. 
normality plots. Non-normally distributed data were transformed into approximately normal distributions, if possible, by logarithmic or square root transformations. The data are appropriately expressed as mean and 95\% CI, if normally distributed; geometric means and 95\% CI, if log-transformed; medians (25th and 75 th percentiles), if non-normally distributed data; or as frequencies.

Changes in plasma lipids and anthropometric measures from baseline 1 to baseline 2 were examined using dependent Student's $t$ tests. The data for the intervention were examined for any interaction effects due to the sequence of intervention (kiwifruit followed by control and vice versa) using two-way ANOVA. If an interaction was seen, only the data from the first 4 weeks of the intervention were analysed. If no interaction was seen, the data from the two intervention periods were combined. Comparisons between baseline 2 and end of the interventions (control and green kiwifruit interventions separately) and between control and kiwifruit interventions for changes in variables were made using dependent Student's $t$ tests. Two-way ANOVA was also used to examine APOE genotype and treatment interactions. However, as the assumptions of homogeneity of variance for ANOVA were not satisfied, lipid results for the two interventions were analysed stratified for APOE4 carriers $v$. non-carriers using dependent Student's $t$ tests and independent Student's $t$ tests. Significance was set at $P<0.05$. All tests were two-tailed.

\section{Results}

\section{Characteristics of subjects}

All eighty-seven men who met the inclusion criteria and were randomly assigned to the two intervention groups completed the 12-week intervention. However, based on their diaries kept during the intervention, two subjects were excluded from the analysis as a result of poor compliance $(<80 \%)$ for the kiwifruit intervention. The flow of subjects through the different phases of the study is illustrated in Fig. 1.

The baseline characteristics of the eighty-five subjects who completed the study are shown in Table 1 .

The men were healthy (no diagnosed chronic disease) and predominantly normotensive, but three-quarters were overweight or obese (BMI $\geq 25 \mathrm{~kg} / \mathrm{m}^{2}$ ) and more than $60 \%$ were carrying excess body fat (percentage of body fat $\geq 25 \%)^{(34,35)}$. Analysis of the $A P O E$ genotype identified twenty-three men $(27 \cdot 4 \%)$ who were carriers of the APOE4 allele (one E2/E4, twenty-one E3/E4 and one E4/E4) and sixty-one (72.6\%) non-carriers (six E2/E3 and fifty-five $E 3 / E 3$ ). One subject requested not to be included in any gene analysis.

\section{Effects of the intervention on dietary intake, body weight and blood pressure}

An assessment of the group's mean energy intake and that of other selected dietary components was made before the nutrition consultation, based on the average intake from a $3 \mathrm{~d}$ food record and during the intervention by using the average of three $24 \mathrm{~h}$ records collected at visits 3-5 (Table 2). Significant differences were seen for all nutrients assessed (except percentage of energy from PUFA) between the two periods, with decreases in total energy and cholesterol intake and the percentage of energy from fat and saturated fat.

During the kiwifruit intervention, the men consumed half a fruit serving per $\mathrm{d}$ more (fresh, canned/stewed, dried and real fruit juice) compared with the control intervention. Significantly higher intakes were seen for vitamins $\mathrm{C}$ and $\mathrm{E}$ during the kiwifruit intervention compared with the control intervention (Table 3).

During the 4-week healthy diet run-in period, small but significant decreases were observed for all anthropometric

Table 2. Composition of the diet pre-nutrition consultation and during the intervention*

(Mean values, geometric means, 95\% confidence intervals, medians, 25th and 75th percentiles, $n$ 85)

\begin{tabular}{|c|c|c|c|c|c|}
\hline & \multicolumn{2}{|c|}{ Pre-consultation } & \multicolumn{2}{|c|}{ During intervention } & \multirow[b]{2}{*}{$P \dagger$} \\
\hline & Mean & $95 \% \mathrm{Cl}$ & Mean & $95 \% \mathrm{Cl}$ & \\
\hline Energy (kJ) & 10623 & 10040,11206 & 9094 & 8694,9494 & $<0.0001$ \\
\hline \multicolumn{5}{|l|}{ Protein (\%E) } & $<0.0001$ \\
\hline Geometric mean & \multicolumn{2}{|r|}{$17 \cdot 8$} & \multirow{2}{*}{\multicolumn{2}{|c|}{$\begin{array}{c}19 \cdot 2 \\
18 \cdot 2\end{array}$}} & \\
\hline $95 \% \mathrm{Cl}$ & \multicolumn{2}{|r|}{$17 \cdot 2,18 \cdot 4$} & & & \\
\hline Carbohydrate (\%E) & 44.6 & $43 \cdot 0,46 \cdot 2$ & $49 \cdot 6$ & $48 \cdot 2,51 \cdot 0$ & $<0.0001$ \\
\hline Fat $(\% \mathrm{E})$ & 32.4 & $31 \cdot 0,33 \cdot 7$ & $27 \cdot 5$ & $26 \cdot 3,28 \cdot 7$ & $<0.0001$ \\
\hline SFA (\%E) & $12 \cdot 8$ & $12 \cdot 0,13 \cdot 5$ & 9.74 & $9 \cdot 13,10 \cdot 4$ & $<0.0001$ \\
\hline MUFA (\%E) & $11 \cdot 3$ & $10 \cdot 8,11.9$ & 9.92 & $9.41,10.4$ & $<0.0001$ \\
\hline \multicolumn{5}{|l|}{ PUFA (\%E) } & 0.74 \\
\hline Geometric mean & \multirow{2}{*}{\multicolumn{2}{|c|}{$\begin{array}{c}4 \cdot 34 \\
4.04,4 \cdot 67\end{array}$}} & \multicolumn{2}{|c|}{4.41} & \\
\hline $95 \% \mathrm{Cl}$ & & & \multicolumn{2}{|c|}{$4 \cdot 14,4 \cdot 69$} & \\
\hline Cholesterol (mg) & \multicolumn{2}{|r|}{$4.04,4.67$} & & & $<0.0001$ \\
\hline Median & \multicolumn{2}{|r|}{328} & \multicolumn{2}{|c|}{239} & \\
\hline 25th-75th percentile & \multicolumn{2}{|r|}{$230-462$} & \multicolumn{2}{|c|}{$174-363$} & \\
\hline
\end{tabular}

$\% \mathrm{E}$, percentage energy of total energy.

*Pre-consultation intake: average of $3 \mathrm{~d}$ food record. During intervention intake: average three $24 \mathrm{~h}$ food records from visits $3-5$.

† Mean values were significantly different between pre-consultation and during intervention $(P<0.05)$ (dependent Student's $t$ test), or Wilcoxon signed ranks test for non-parametric data $(P<0.05)$. 
measures (body weight, BMI, waist circumference, waist:hip ratio and percentage body fat). From the end of the run-in period (baseline 2) to the end of the 8-week intervention, there were no further changes in body weight, regardless of which intervention the subjects were receiving (green kiwifruit or control) (Table 4).

There were no significant changes in systolic or diastolic blood pressure (Table 4).

\section{Tolerance to the kiwifruit intervention}

The majority of subjects rated eating two green kiwifruits every day as easy or very easy at both the beginning $(78.8 \%)$ and end (89.4\%) of the intervention. A total of seventy-five subjects $(88 \%)$ reported no unwanted side effects from eating two green kiwifruits per d. The most commonly reported unwanted side-effect was more frequent or looser bowel motions (3.5\%).

\section{Nutrient composition of the green kiwifruit}

Two green kiwifruits, without their skin, weighed on average $167 \mathrm{~g}$ and provided on average $1.58 \mathrm{~g}$ of protein, $21.9 \mathrm{~g}$ carbohydrate, $14.9 \mathrm{~g}$ sugars, $130 \mathrm{mg}$ of vitamin $\mathrm{C}, 1.00 \mathrm{mg}$ vitamin $\mathrm{E}$, $4.11 \mathrm{~g}$ insoluble fibre and $1.42 \mathrm{~g}$ soluble fibre.

\section{Effects on plasma lipid and apo concentrations}

Significant improvements were seen in lipid profiles following the 4-week run-in period (Table 5), with both plasma TC and LDL-C decreasing and HDL-C increasing significantly $(P<0.05)$. There was also a non-significant trend towards a decrease in plasma TAG.

No significant differences between the two interventions were seen for plasma TC, LDL-C, TAG and sLDL. There was a significant increase in HDL-C concentrations while consuming green kiwifruit compared with the control (mean difference $0.04 ; 95 \%$ CI $0.01,0.07 \mathrm{mmol} / 1 ; P=0.004)$, with TC:HDL-C ratio significantly lower after the green kiwifruit period (mean difference $-0.15 ; 95 \%$ CI $-0.24,-0.05 \mathrm{mmol} / 1$; $P=0 \cdot 002)$. Serum apoA1 concentrations increased significantly from baseline 2 to end of the green kiwifruit intervention (mean difference $0.03 ; 95 \% \mathrm{CI} 0.001 \mathrm{~g} / 1,0.06 ; P=0.03$ ), resulting in a decrease in the apoB:apoA1 ratio with green kiwifruit consumption compared with control (although not significant, $P=0.05)$.

\section{APOE genotype-kiwifruit treatment interaction}

There were no significant differences between $A P O E 4$ carriers and non-carriers at baseline for any anthropometric measures, blood pressure, hs-CRP, HOMA2-insulin resistance (data not shown) or lipid concentrations (Table 6). Significant improvements in TC, LDL-C and HDL-C were seen in the non-carriers of $A P O E 4$ in response to the 4-week healthy diet period $(P<0.05)$. The same trends and magnitude of change were seen for the APOE4 carriers, but the changes were not 
Table 4. Anthropometric and blood pressure assessments: baseline 1 to baseline 2 , and after the two intervention periods* (Mean values and $95 \%$ confidence intervals, $n 85$ )

\begin{tabular}{|c|c|c|c|c|c|c|c|c|c|c|}
\hline \multirow[b]{2}{*}{ Variable } & \multicolumn{2}{|c|}{ Baseline 1} & \multicolumn{2}{|c|}{ Baseline 2} & \multirow[b]{2}{*}{$P \dagger$} & \multicolumn{2}{|c|}{ End-kiwifruit } & \multicolumn{2}{|c|}{ End-control } & \multirow[b]{2}{*}{$P \ddagger$} \\
\hline & Mean & $95 \% \mathrm{Cl}$ & Mean & $95 \% \mathrm{Cl}$ & & Mean & $95 \% \mathrm{Cl}$ & Mean & $95 \% \mathrm{Cl}$ & \\
\hline Weight (kg) & $86 \cdot 7$ & $83 \cdot 3,90 \cdot 2$ & $86 \cdot 4$ & $83 \cdot 0,89 \cdot 8$ & 0.03 & $86 \cdot 7$ & $83 \cdot 3,90 \cdot 0$ & $86 \cdot 6$ & $83 \cdot 2,90 \cdot 0$ & 0.86 \\
\hline BMI $\left(\mathrm{kg} / \mathrm{m}^{2}\right)$ & $27 \cdot 4$ & $26 \cdot 5,28 \cdot 2$ & $27 \cdot 3$ & $26 \cdot 5,28 \cdot 1$ & 0.03 & $27 \cdot 3$ & $26 \cdot 5,28 \cdot 2$ & $27 \cdot 3$ & $26 \cdot 5,28 \cdot 2$ & 0.87 \\
\hline Waist $(\mathrm{cm})$ & 93.4 & $91 \cdot 3,95 \cdot 6$ & $93 \cdot 1$ & $90 \cdot 9,95 \cdot 3$ & 0.03 & $92 \cdot 8$ & $90 \cdot 7,94.9$ & 93.0 & $90 \cdot 9,95 \cdot 2$ & 0.31 \\
\hline Waist:hip ratio & 0.91 & $0.90,0.92$ & 0.90 & $0.89,0.92$ & 0.001 & 0.90 & $0.89,0.91$ & 0.90 & $0.89,0.91$ & 0.39 \\
\hline Body fat (\%) & $27 \cdot 4$ & $25 \cdot 8,29 \cdot 0$ & $26 \cdot 9$ & $25 \cdot 2,28 \cdot 6$ & 0.002 & $26 \cdot 9$ & $25 \cdot 2,28 \cdot 6$ & $26 \cdot 8$ & $25 \cdot 2,28 \cdot 5$ & 0.53 \\
\hline $\mathrm{SBP}(\mathrm{mmHg})$ & & NA & 122 & 120,125 & & 124 & 122,127 & 124 & 122,127 & 0.90 \\
\hline $\mathrm{DBP}(\mathrm{mmHg})$ & & NA & 70.9 & $69 \cdot 0,72 \cdot 8$ & & $72 \cdot 3$ & $70 \cdot 4,74 \cdot 3$ & $72 \cdot 6$ & $70 \cdot 4,74 \cdot 7$ & 0.80 \\
\hline
\end{tabular}

Baseline 1, before run-in period; baseline 2, after run-in period; SBP, systolic blood pressure; NA, not assessed; DBP, diastolic blood pressure.

* Subjects followed a cross-over design protocol for the two intervention periods.

† Mean values were significantly different from baseline 1 to baseline $2(P<0.05$; dependent Student's $t$ test).

$\ddagger$ Mean values were significantly different between green kiwifruit $v$. control $(P<0.05$; dependent Student's $t$ test).

significant, which is probably reflective of the smaller sample size in the APOE4 carriers' subgroup.

The most significant difference seen between APOE4 carriers and non-carriers in response to the two interventions was the effects on TAG concentrations. APOE4 carriers showed a significant decrease in TAG concentrations following the kiwifruit intervention compared with the control, resulting in a significant difference between the two interventions (mean difference $-0.18 ; 95 \% \mathrm{CI}-0.34,-0.02 \mathrm{mmol} / \mathrm{l}$; $P=0.03$ ). This also resulted in a significant difference in TAG concentrations between APOE4 carriers and non-carriers for the kiwifruit intervention (mean difference $1.34,95 \% \mathrm{CI}$ $1 \cdot 14,1.54 \mathrm{mmol} / 1$ v. $1 \cdot 66,95 \%$ CI $1.50,1 \cdot 85 \mathrm{mmol} / 1 ; P=0 \cdot 01)$.

In non-carriers, there was a significant difference between kiwifruit and control for plasma HDL-C and the TC:HDL-C ratio. This was largely due to the significant decrease in HDL-C and increase in the TC:HDL-C ratio for the control intervention from baseline 2. In contrast, while not significant, APOE4 carriers had an increase in HDL-C from baseline 2 $(P=0 \cdot 14)$. Serum apoA1 concentration increased in the $A P O E 4$ non-carriers during the green kiwifruit period, which was reflected in the apoB:apoA1 ratio $(P=0.03)$.

\section{Other CVD-related markers}

There were no significant changes in hs-CRP, plasma insulin, glucose or HOMA2-insulin resistance, between the two interventions (Tables S1 and S2, available online).

\section{Discussion}

In the present study, consumption of two green kiwifruits a day for 4 weeks had favourable effects on plasma HDL-C and the TC:HDL-C ratio compared with a healthy control diet. The hypotriacylglycerolaemic impact of the intervention was only evident in APOE4 carriers.

A large proportion $(60 \%)$ of men did not meet the recommended two servings of fruit per $\mathrm{d}$ at the start of the study, compared with $45 \%$ men from the latest (2008/2009) NZ Adult Nutrition survey, even when including dried fruit and real fruit juice (not included in the nutrition survey) ${ }^{(4)}$. The median number of fruit servings per $d$ increased from $1 \cdot 3(25$ th -75 th percentiles $0 \cdot 7-2 \cdot 7)$ at baseline to $3 \cdot 0$ (25th-75th percentiles $3 \cdot 0-4 \cdot 0$ ) during kiwifruit and $2 \cdot 5$ (25th-75th percentiles $2 \cdot 0-4 \cdot 0$ ) during control interventions. Although the aim was to replace two fruit servings with two kiwifruits, half a serving more of fruit was consumed during the kiwifruit intervention compared with the control period. The five most commonly consumed other fruits were apples, bananas, oranges, pears and mandarins.

The 4-week run-in period on the healthy dietary recommendations resulted in significant improvements in all measured lipid parameters, except TAG, which approached significance. This confirms the efficacy of the present NZ Heart Foundation's guidelines.

Two green kiwifruits per d did not affect LDL-C compared with the control diet. Brown et al. ${ }^{(8)}$ showed that for every $1 \mathrm{~g}$ increase in soluble fibre, LDL-C decreased by $0.06 \mathrm{mmol} / \mathrm{l}$. The $1.8 \mathrm{~g} / \mathrm{d}$ difference in total fibre (including insoluble plus soluble fibre) intake between the kiwifruit and control interventions may not have been sufficient to affect LDL-C concentrations.

The green kiwifruit intervention resulted in a significantly improved mean HDL-C concentration and TC:HDL-C ratio compared with the control intervention. Adding strength to these findings, the green kiwifruit intervention significantly increased apoA1 (the main structural protein component of HDL) concentrations from baseline 2 and consequently contributed to the almost significantly lower apoB:A1 ratio $(P=0.05)$, compared with the control intervention. It has been suggested that for every $0.1 \mathrm{mmol} / 1$ increase in HDL-C, the CHD risk would reduce by between 8 and $15 \%{ }^{(36,37)}$; therefore, the difference seen in the present study could be translated into a 3-6\% reduction in risk. These findings are in line with that of Chang \& $\mathrm{Liu}^{(16)}$, who showed that consumption of two green kiwifruits per d for 8 weeks significantly increased HDL-C concentration and decreased the TC:HDL-C ratio compared with the baseline levels. In contrast, Duttaroy \& Jorgensen ${ }^{(17)}$ found no effect on HDL-C. Similar to kiwifruit, berries contain vitamins $\mathrm{C}$ and $\mathrm{E}$ and folic acid, and are a good source of carotenoids and other polyphenols ${ }^{(38)}$. An 8-week randomised, controlled berry intervention has also shown significant improvements in HDL-C concentrations compared with control products ${ }^{(39)}$. 

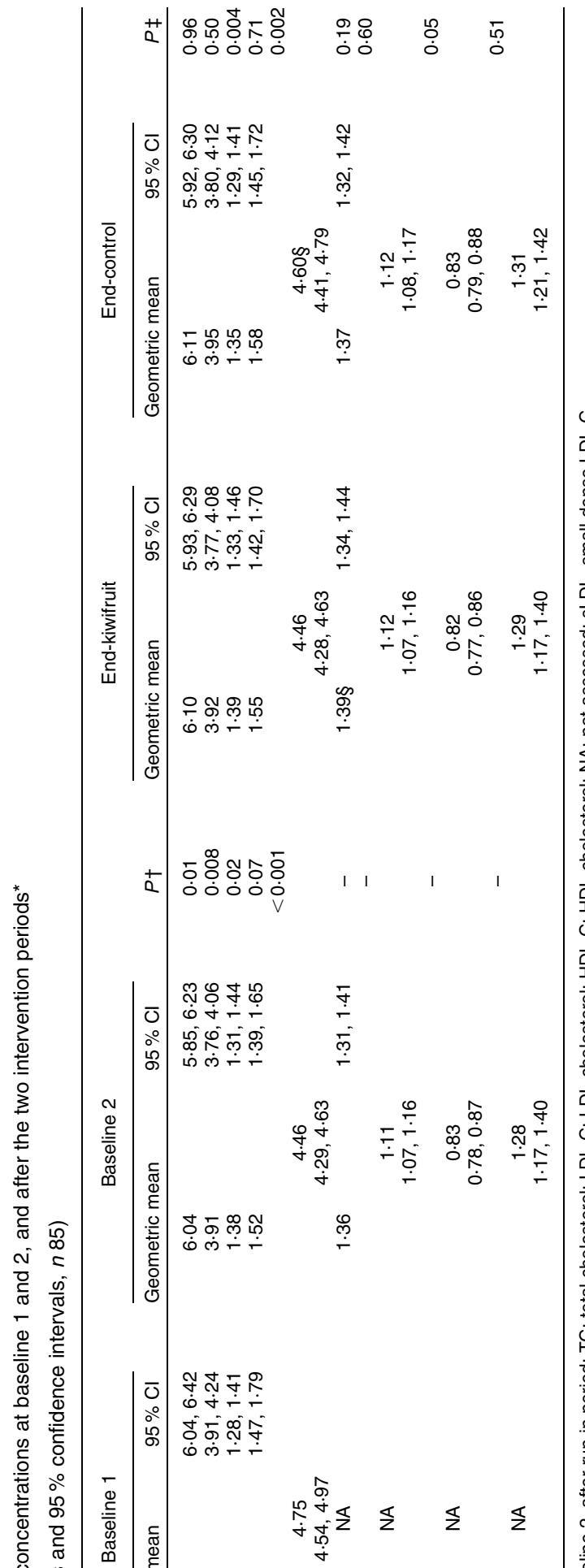

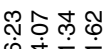

The most likely constituent to exert the observed effects are polyphenols. Flavonoid-rich juices such as orange juice ${ }^{(40)}$, grape juice ${ }^{(41)}$ and cranberry juice ${ }^{(42)}$ have all been shown to increase plasma HDL-C. While components other than polyphenols, such as vitamin $\mathrm{C}$ in fruit and juices, cannot be discounted, polyphenols from cocoa have also shown HDLC-raising effects ${ }^{(43,44)}$. Various mechanisms have been proposed, but in one recent cell study it was shown that cocoa polyphenols increased apoA1 production ${ }^{(45)}$.

Circulating TAG is metabolically intimately linked with HDL, with elevated TAG levels resulting in increased hepatic lipasemediated HDL hydrolysis and decreased HDL-C concentration ${ }^{(46)}$. Lowering TAG concentrations have been shown to increase HDL-C in both normo- and hypertriacylglycerolaemic subjects ${ }^{(47)}$. Chang \& $\mathrm{Liu}^{(16)}$ showed a trend towards a decrease in TAG, as did some of the flavonoid-rich juice studies mentioned earlier ${ }^{(41,42)}$. The present study showed no significant change in TAG concentrations for the complete group. However, in APOE4 carriers, plasma TAG concentrations were significantly lowered after consumption of green kiwifruit.

Duttaroy \& Jorgensen ${ }^{(17)}$ showed that plasma TAG were significantly reduced by $15 \%$ compared with the baseline values following kiwifruit consumption, as did a more recent study examining the effects of kiwifruit on biomarkers of oxidative status ${ }^{(48)}$. Both publications offered little explanation beyond that such effects have been seen before with fruits and vegetables.

A recent placebo-controlled trial in subjects who received $120 \mathrm{mg}$ gamma delta tocotrienols per d showed a $28 \%$ reduction in serum $\mathrm{TAG}^{(49)}$. Despite the dose being higher than is found naturally in fruit, it does hint at the possibility that a component such as this, working synergistically with other bioactive components, may affect TAG concentrations. Polyphenols could also explain the decrease, with studies with red grapefruit and tomatoes also showing positive effects on plasma TAG concentrations ${ }^{(50,51)}$. Interestingly, in the tomato study, the positive effects were seen with tomato juice and whole fruit, but not with the lycopene drink ${ }^{(50)}$.

APOE4 carriers appear to be the most responsive to reduced SFA, total fat and cholesterol intake, with reductions seen in TC and LDL-C ${ }^{(21)}$. One study showed a greater responsiveness of APOE4 carriers to the TAG-lowering effect of fish oils ${ }^{(52)}$. To the best of our knowledge, the present study is the first to show a different response to eating fruit on TAG in $A P O E 4$ carriers. It has been suggested that APOE4 represents a 'lipid-thrifty' variant, which allows better intestinal absorption of lipids, including fat-soluble vitamins (and polyphenols $)^{(53-55)}$. Thus, it could be speculated that the absorption of vitamin E, carotenoids and polyphenols from kiwifruit may be enhanced in APOE4 carriers, resulting in a TAGlowering effect.

Given the chemically complex composition of kiwifruit and the opportunity for both independent and/or synergistic contributions of constituents in biological processes, the complete elucidation of the mechanism to explain the observed increase in HDL-C and decrease in TAG in APOE4 carriers remains to be determined. 
Table 6. The impact of $A P O E$ genotype on lipid and apo concentrations at baseline 1 and 2 , and after the two intervention periods ${ }^{*}$

(Mean values, geometric means and $95 \%$ confidence intervals; $n 23$ APOE4 carriers and $n 61$ APOE4 non-carriers)

\begin{tabular}{|c|c|c|c|c|c|c|c|c|c|c|}
\hline & \multicolumn{2}{|c|}{ Baseline 1} & \multicolumn{2}{|c|}{ Baseline 2} & \multirow[b]{2}{*}{$P \dagger$} & \multicolumn{2}{|c|}{ End-kiwifruit } & \multicolumn{2}{|c|}{ End-control } & \multirow[b]{2}{*}{$P \ddagger$} \\
\hline & Geometric mea & $95 \% \mathrm{Cl}$ & Geometric mean & $95 \% \mathrm{Cl}$ & & Geometric mean & $95 \% \mathrm{Cl}$ & Geometric mean & $95 \% \mathrm{Cl}$ & \\
\hline \multicolumn{11}{|l|}{ TC $(\mathrm{mmol} / \mathrm{l})$} \\
\hline APOE 4 carriers & $6 \cdot 38$ & $5.95,6.84$ & $6 \cdot 18$ & $5 \cdot 75,6.65$ & 0.30 & $6 \cdot 31$ & $5 \cdot 89,6 \cdot 74$ & $6 \cdot 33$ & $5.92,6.77$ & 0.84 \\
\hline APOE4 non-carriers & $6 \cdot 18$ & $5.97,6.40$ & 6.00 & $5.79,6.22$ & 0.02 & 6.03 & $5.84,6.24$ & 6.02 & $5 \cdot 81,6 \cdot 25$ & 0.87 \\
\hline \multicolumn{11}{|l|}{ LDL-C (mmol/l) } \\
\hline APOE 4 carriers & & & & & 0.38 & & & & & 0.91 \\
\hline Mean & \multirow{2}{*}{\multicolumn{2}{|c|}{$\begin{array}{c}4 \cdot 25 \\
3 \cdot 84,4 \cdot 66\end{array}$}} & \multirow{2}{*}{\multicolumn{2}{|c|}{$\begin{array}{c}4 \cdot 11 \\
3.70,4 \cdot 52\end{array}$}} & & \multirow{2}{*}{\multicolumn{2}{|c|}{$\begin{array}{c}4 \cdot 25 \S \\
3.90,4 \cdot 60\end{array}$}} & \multicolumn{2}{|c|}{4.24} & \\
\hline $95 \% \mathrm{Cl}$ & & & & & & & & \multirow{2}{*}{\multicolumn{2}{|c|}{$3.89,4.59$}} & \\
\hline APOE4 non-carriers & \multicolumn{2}{|c|}{ (2) } & \multirow{3}{*}{\multicolumn{2}{|c|}{$\begin{array}{c}3.92 \\
3.76,4.09\end{array}$}} & 0.01 & \multirow{3}{*}{\multicolumn{2}{|c|}{$\begin{array}{c}3.89 \S \\
3.71,4 \cdot 07\end{array}$}} & & & 0.33 \\
\hline Mean & \multirow{2}{*}{\multicolumn{2}{|c|}{$\begin{array}{c}4 \cdot 11 \\
3 \cdot 91,4 \cdot 30\end{array}$}} & & & & & & \multirow{2}{*}{\multicolumn{2}{|c|}{$\begin{array}{c}3.95 \\
3.76,4 \cdot 14\end{array}$}} & \\
\hline $95 \% \mathrm{Cl}$ & & & & & & & & & & \\
\hline HDL-C (mmol/l) & & & & & & \multicolumn{2}{|c|}{$3 \cdot 71,4.07$} & & & \\
\hline APOE4 carriers & 1.41 & $1.28,1.57$ & 1.43 & $1.28,1.60$ & 0.58 & 1.48 & $1.34,1.64$ & 1.45 & $1.30,1 \cdot 61$ & 0.29 \\
\hline$A P O E 4$ non-carriers & 1.32 & $1.25,1.39$ & 1.36 & $1.29,1.43$ & 0.02 & 1.37 & $1.30,1.44$ & $1.32 \|$ & $1.25,1.38$ & 0.003 \\
\hline TAG $(\mathrm{mmol} / \mathrm{l})$ & & & & & & & & & & \\
\hline APOE 4 carriers & & & & & $0 \cdot 14$ & & & & & 0.03 \\
\hline $\begin{array}{l}\text { Mean } \\
95 \% \mathrm{Cl}\end{array}$ & $\begin{array}{r}1 \\
1.2 S^{2} \\
\end{array}$ & & $\begin{array}{r}1 \\
1.21\end{array}$ & & & $\begin{array}{r}1 \cdot \\
1 \cdot 14\end{array}$ & & $\begin{array}{r}1 . \\
1.31\end{array}$ & & \\
\hline APOE4 non-carriers & 1.66 & $1.48,1.86$ & 1.56 & $1.42,1.72$ & 0.19 & $1.66 \S$ & $1.50,1.85$ & 1.62 & $1.46,1.80$ & 0.61 \\
\hline TC:HDL-C ratio & & & & & & & & & & \\
\hline APOE4 carriers & & & & & 0.05 & & & & & 0.15 \\
\hline $\begin{array}{l}\text { Mean } \\
95 \% \mathrm{Cl}\end{array}$ & & & 4 & & & 4 & & 4 & & \\
\hline $\begin{array}{l}95 \% \mathrm{Cl} \\
\text { APOE4 non-carriers }\end{array}$ & $4 \cdot 17$ & & 4.05 & & & 3.99 & & 4.10, & & \\
\hline $\begin{array}{l}\text { APOE } 4 \text { non-carriers } \\
\text { Mean }\end{array}$ & & & & & $<0.001$ & & & & & 0.005 \\
\hline $95 \% \mathrm{Cl}$ & $\begin{array}{r}4 \\
4.53\end{array}$ & & $\begin{array}{r}4 \\
4.28\end{array}$ & & & $\begin{array}{r}4 \\
4.28\end{array}$ & & $4 \cdot 6$ & & \\
\hline ApoA1 (g/l) & & & $4 \cdot \angle 8$ & & & $4 \cdot \angle 8$ & & 4.43, & & \\
\hline APOE4 carriers & & & 1.41 & $1.30,1.53$ & - & 1.43 & $1.32,1.56$ & 1.44 & $1.32,1.57$ & 0.80 \\
\hline APOE4 non-carriers & & & 1.35 & $1.29,1.40$ & - & $1.38 \|$ & $1.33,1.44$ & 1.35 & $1.30,1.41$ & 0.05 \\
\hline $\mathrm{ApoB}(\mathrm{g} / \mathrm{l})$ & & & & & & & & & & \\
\hline APOE4 carriers & & & & & - & & & & & 0.74 \\
\hline $\begin{array}{l}\text { Mean } \\
95 \% \mathrm{Cl}\end{array}$ & & & $\begin{array}{r}1 \\
1.02\end{array}$ & & & $\begin{array}{r}1 \\
1.03\end{array}$ & & $\begin{array}{r}1 . \\
1.05\end{array}$ & & \\
\hline APOE4 non-carriers & & & & & - & & & & & 0.75 \\
\hline Mean & & & 1 & & & 1 & & 1. & & \\
\hline $\begin{array}{c}95 \% \mathrm{Cl} \\
\text { ApoB:apoA1 ratio }\end{array}$ & & & 1.06 & & & 1.06 & & 1.06, & & \\
\hline $\begin{array}{l}\text { ApoB:apoA1 ratio } \\
\text { APOE4 carriers }\end{array}$ & & & & & - & & & & & 0.80 \\
\hline Mean & & & 0 & & & 0 & & 0 & & \\
\hline $95 \% \mathrm{Cl}$ & & & 0.71 & & & 0.71 & & 0.72 & & \\
\hline$A P O E 4$ non-carriers & & & & & - & & & & & 0.03 \\
\hline Mean & & & 0 & & & 0 & & 0 & & \\
\hline $95 \% \mathrm{Cl}$ & & & 0.78 & & & 0.76 & & 0.78 & & \\
\hline $\mathrm{sLDL}(\mathrm{mmol} / \mathrm{l})$ & & & & & & & & & & \\
\hline APOE4 carriers & & & & & & & & & & 0.49 \\
\hline Mean & & & 1 & & & 1 & & 1. & & \\
\hline $95 \% \mathrm{Cl}$ & & & 1.03 & & & 1.08 & & 1.15, & & \\
\hline APOE4 non-carriers & & & & & & & & & & 0.71 \\
\hline Mean & & & 1 & & & 1 & & 1. & & \\
\hline $95 \% \mathrm{Cl}$ & & & $1 \cdot 15$ & & & $1 \cdot 13$ & & 1.16, & & \\
\hline
\end{tabular}

Baseline 1, before run-in period; baseline 2, after run-in period; TC, total cholesterol; LDL-C, LDL-cholesterol; HDL-C, HDL-cholesterol; NA, not assessed; sLDL, small dense LDL-C.

"Subjects followed a cross-over design protocol for the two intervention periods. No interaction effects were seen between sequence of intervention and intervention (two-way ANOVA)

† Mean values were significantly different from baseline 1 to baseline $2(P<0.05$; dependent Student's $t$ test).

¥ Mean values were significantly different between green kiwifuit $v$. control $(P<0.05$; dependent Student's $t$ test $)$

$\S$ Mean values were significantly different between APOE 4 carriers and non-carriers $(P<0.05$; independent Student's $t$ test).

Mean values were significantly different from baseline 2 to end $(P<0.05$; dependent Student's $t$ test). 
Considering that benefits on plasma TAG and HDL-C were evident, which represent core components of the dyslipidaemia associated with obesity and loss of insulin sensitivity, further work to identify the bioactive constituents and the mechanisms underlying their observed benefits is merited.

An unavoidable limitation of the present study is that the use of a fruit intervention precludes the use of a doubleblind study design. A further limitation is that the results cannot be extrapolated to women. Strengths include the randomised cross-over design with the 4-week run-in period, wide age and BMI ranges, the inclusion of APOE4 subgroup analysis and a large sample size with sufficient statistical power for APOE4 subgroup analysis.

In conclusion, we found that consuming two green kiwifruits per d showed improvement (although modest) in the CVD risk profile against an overall healthy diet background, with some indication that the APOE4 (approximately $25 \%$ of the population) subgroup may be most responsive.

\section{Supplementary material}

To view supplementary material for this article, please visit http://dx.doi.org/10.1017/S0007114512004400

\section{Acknowledgements}

The present study was funded by an unrestricted grant from Zespri $^{\circledR}$ International Limited, NZ. The authors have no conflicts of interest to declare. W. S., R. K., C. A. C., P. R. v. H. and C. S. G. were involved in study design and data collection; C. S. G. and W. S. carried out the statistical analysis; A. M. M. analysed $A P O E$ data and conducted SLDL analysis; C. S. G. wrote the initial draft of the manuscript, which was modified after feedback from all co-authors. We would like to thank the subjects who participated in the study and the IFNHH research team, with special thanks to Olivia Green, Regina Wypych and Simon Bennett for their help with data collection and Professor John Birkbeck for counselling men identified with the APOE4 genotype.

\section{References}

1. Ministry of Health (2011) Mortality and Demographic Data 2008. Wellington: Ministry of Health.

2. Tobias M, Sexton K, Mann S, et al. (2006) How low can it go? Projecting ischaemic heart disease mortality in New Zealand to 2015. N Z Med J 119, U1932.

3. Tobias M, Taylor R, Yeh L-C, et al. (2008) Did it fall or was it pushed? The contribution of trends in established risk factors to the decline in premature coronary heart disease mortality in New Zealand. Aust N Z J Public Health 32, 117-125.

4. University of Otago and Ministry of Health (2011) A Focus on Nutrition: Key Findings of the 2008/09 New Zealand Adult Nutrition Survey. Wellington: Ministry of Health.

5. Badimon L, Vilahur G \& Padro T (2010) Nutraceuticals and atherosclerosis: human trials. Cardiovasc Ther 28, 202-215.

6. Voutilainen S, Nurmi T, Mursu J, et al. (2006) Carotenoids and cardiovascular health. Am J Clin Nutr 83, 1265-1271.

7. World Health Organization (2003) Diet, Nutrition and the Prevention of Chronic Diseases. Joint WHO/FAO Expert
Consultation. WHO Technical Report Series no. 916 Geneva: WHO.

8. Brown L, Rosner B, Willett WW, et al. (1999) Cholesterollowering effects of dietary fiber: a meta-analysis. Am J Clin Nutr 69, 30-42

9. Erkkila AT \& Lichtenstein AH (2006) Fiber and cardiovascular disease risk: how strong is the evidence? J Cardiovasc Nurs 21, 3-8.

10. Salas-Salvado J, Bullo M, Perez-Heras A, et al. (2006) Dietary fibre, nuts and cardiovascular diseases. Br J Nutr 96 S46-S51.

11. Schakel S, Pettit J \& Himes H (2001) Dietary fiber values for common foods. In The CRC Handbook of Dietary Fiber in Human Nutrition, 3rd ed. [G Spiller, editor]. London: CRC Press.

12. Ferguson AR \& Ferguson LR (2003) Are kiwifruit really good for you? Acta Hortic 610, 131-135.

13. Vissers MCM, Bozonet SM, Pearson JF, et al. (2011) Dietary ascorbate intake affects steady state tissue concentrations in vitamin C-deficient mice: tissue deficiency after suboptimal intake and superior bioavailability from a food source (kiwifruit). Am J Clin Nutr 93, 292-301.

14. Hunter DC, Greenwood J, Zhang J, et al. (2011) Antioxidant and 'natural protective' properties of kiwifruit. Curr Top Med Chem 11, 1811-1820.

15. Tarascou I, Souquet JM, Mazauric JP, et al. (2010) The hidden face of food phenolic composition. Arch Biochem Biophys 501, 16-22.

16. Chang WH \& Liu JF (2009) Effects of kiwifruit consumption on serum lipid profiles and antioxidative status in hyperlipidemic subjects. Int J Food Sci Nutr 60, 709-716.

17. Duttaroy AK \& Jorgensen A (2004) Effects of kiwi fruit consumption on platelet aggregation and plasma lipids in healthy human volunteers. Platelets 15, 287-292.

18. Karlsen A, Svendsen M \& Seljeflot I, et al. (2012) Kiwifruit decreases blood pressure and whole-blood platelet aggregation in male smokers J Hum Hypertens (Epublication ahead of print version 19 January 2012).

19. Elder SJ, Lichtenstein AH, Pittas AG, et al. (2009) Genetic and environmental influences on factors associated with cardiovascular disease and the metabolic syndrome. J Lipid Res 50, 1917-1926.

20. Corella D \& Ordovas JM (2005) Single nucleotide polymorphisms that influence lipid metabolism: interaction with dietary factors. Annu Rev Nutr 25, 341-390.

21. Masson LF, McNeill G \& Avenell A (2003) Genetic variation and the lipid response to dietary intervention: a systematic review. Am J Clin Nutr 77, 1098-1111.

22. Song Y, Stampfer MJ \& Liu S (2004) Meta-analysis: apolipoprotein $\mathrm{E}$ genotypes and risk for coronary heart disease. Ann Intern Med 141, 137-147.

23. Jofre-Monseny L, Minihane AM \& Rimbach G (2008) Impact of apoE genotype on oxidative stress, inflammation and disease risk. Mol Nutr Food Res 52, 131-145.

24. Bennet AM, Di Angelantonio E, Ye Z, et al. (2007) Association of apolipoprotein $\mathrm{E}$ genotypes with lipid levels and coronary risk. JAMA 298, 1300-1311.

25. Rimbach G \& Minihane AM (2009) Nutrigenetics and personalised nutrition: how far have we progressed and are we likely to get there? Proc Nutr Soc 68, 162-172.

26. de Sauvage Nolting PR, Buirma RJ, Hutten BA, et al. (2002) Baseline lipid values partly determine the response to high-dose simvastatin in patients with familial hypercholesterolemia. The examination of probands and relatives in Statin studies with familial hypercholesterolemia. Atherosclerosis 164, 347-354 
27. Gould AL, Davies GM, Alemao E, et al. (2007) Cholesterol reduction yields clinical benefits: meta-analysis including recent trials. Clin Ther 29, 778-794.

28. Heart Foundation of New Zealand (2009) Healthy Heart Eating. http://www.heartfoundation.org.nz/uploads/A\%20 Guide $\% 20$ to\%20Heart\%20Healthy\%20Eating\%202010(4).pdf (accessed March 2010).

29. Friedewald WT, Levy RI \& Fredrickson DS (1972) Estimation of the concentration of low-density lipoprotein cholesterol in plasma, without use of the preparative ultracentrifuge. Clin Chem 18, 499-502.

30. Wallace TM, Levy JC \& Matthews DR (2004) Use and abuse of HOMA modeling. Diabetes Care 27, 1487-1495.

31. Hixson JE \& Vernier DT (1990) Restriction isotyping of human apolipoprotein $\mathrm{E}$ by gene amplification and cleavage with HhaI. J Lipid Res 31, 545-548.

32. Hall MB, Hoover WH, Jennings JP, et al. (1999) A method for partitioning neutral detergent-soluble carbohydrates. J Sci Food Agric 79, 2079-2086.

33. Lee H \& Coates G (1987) Liquid chromatographic determination of vitamin $\mathrm{C}$ in commercial Florida citrus juice. J Micronutr Anal 3, 199-209.

34. Lee R \& Nieman D (2010) Nutritional Assessment, 5th ed. New York, NY: McGraw-Hill.

35. World Health Organization (1995) Physical Status: The Use and Interpretation of Anthropometry. World Health Organization Technical Report Series no. 854. Geneva: WHO.

36. Gordon DJ, Probstfield JL, Garrison RJ, et al. (1989) Highdensity lipoprotein cholesterol and cardiovascular disease. Four prospective American studies. Circulation 79, 8-15.

37. Turner RC, Millns H, Neil HA, et al. (1998) Risk factors for coronary artery disease in non-insulin dependent diabetes mellitus: United Kingdom Prospective Diabetes Study (UKPDS: 23). BMJ 316, 823-828.

38. Basu A, Rhone M \& Lyons TJ (2010) Berries: emerging impact on cardiovascular health. Nutr Rev 68, 168-177.

39. Erlund I, Koli R, Alfthan G, et al. (2008) Favorable effects of berry consumption on platelet function, blood pressure, and HDL cholesterol. Am J Clin Nutr 87, 323-331.

40. Kurowska EM, Spence JD, Jordan J, et al. (2000) HDLcholesterol-raising effect of orange juice in subjects with hypercholesterolemia. Am J Clin Nutr 72, 1095-1100.

41. Albers AR, Varghese S, Vitseva O, et al. (2004) The antiinflammatory effects of purple grape juice consumption in subjects with stable coronary artery disease. Arterioscler Thromb Vasc Biol 24, e179-e180.

42. Ruel G, Pomerleau S, Couture P, et al. (2006) Favourable impact of low-calorie cranberry juice consumption on plasma HDL-cholesterol concentrations in men. Br J Nutr 96, 357-364.
43. Khan N, Monagas M \& Andres-Lacueva C, et al. (2011) Regular consumption of cocoa powder with milk increases HDL-cholesterol and reduces oxidized LDL levels in subjects at high-risk of cardiovascular disease Nutr Metab Cardiovasc Dis (Epublication ahead of print version 5 May 2011).

44. Mellor DD, Sathyapalan T, Kilpatrick ES, et al. (2010) Highcocoa polyphenol-rich chocolate improves HDL-cholesterol in Type 2 diabetes patients. Diabet Med 27, 1318-1321.

45. Yasuda A, Natsume M, Osakabe N, et al. (2011) Cacao polyphenols influence the regulation of apolipoprotein in HepG2 and Caco 2 cells. J Agric Food Chem 59, 1470-1476.

46. Lamarche B, Rashid S \& Lewis GF (1999) HDL metabolism in hypertriglyceridemic states: an overview. Clin Chim Acta 286, 145-161.

47. Miller M, Langenberg P \& Havas S (2007) Impact of lowering triglycerides on raising HDL-C in hypertriglyceridemic and non-hypertriglyceridemic subjects. Int $J$ Cardiol 119, 192-195.

48. Brevik A, Gaivao I, Medin T, et al. (2011) Supplementation of a western diet with golden kiwifruits (Actinidia chinensis var.'Hort 16A':) effects on biomarkers of oxidation damage and antioxidant protection. Nutr J 10, 54 .

49. Zaiden N, Yap WN, Ong S, et al. (2010) Gamma delta tocotrienols reduce hepatic triglyceride synthesis and VLDL secretion. J Atheroscler Thromb 17, 1019-1032.

50. Shen Y-C, Chen S-L \& Wang C-K (2007) Contribution of tomato phenolics to antioxidation and down-regulation of blood lipids. J Agric Food Chem 55, 6475-6481.

51. Gorinstein S, Caspi A, Libman I, et al. (2006) Red grapefruit positively influences serum triglyceride level in patients suffering from coronary atherosclerosis: studies in vitro and in humans. J Agric Food Chem 54, 1887-1892.

52. Caslake MJ, Miles EA, Kofler BM, et al. (2008) Effect of sex and genotype on cardiovascular biomarker response to fish oils: the FINGEN Study. Am J Clin Nutr 88, 618-629.

53. Corbo RM \& Scacchi R (1999) Apolipoprotein E (APOE) allele distribution in the world. Is APOE* 4 a 'thrifty' allele? Ann Hum Genet 63, 301-310.

54. Gerdes LU (2003) The common polymorphism of apolipoprotein E: geographical aspects and new pathophysiological relations. Clin Chem Lab Med 41, 628-631.

55. Huebbe P, Nebel A, Siegert S, et al. (2011) APOE epsilon4 is associated with higher vitamin $\mathrm{D}$ levels in targeted replacement mice and humans. FASEB J 25, 3262-3270.

56. National Cholesterol Education Program (NCEP) Expert Panel (2002) Third Report of the National Cholesterol Education Program (NCEP) Expert Panel on Detection, Evaluation, and Treatment of High Blood Cholesterol in Adults (Adult Treatment Panel III) final report. Circulation 106, 3143-3421. 